Series A

I. MATHEMATICA

332

\title{
ÜBER DAS EXISTENZGEBIET VON INTEGRALEN PARTIELLER DIFFERENTIALGLEICHUNGEN ERSTER ORDNUNG
}

VON

JOUKO MANNINEN 
Am 11 Januar 1963 vorgelegt von R. Nevanlinna und Oldi Lehto 


\section{Über das Existenzgebiet von Integralen partieller Differentialgleichungen erster Ordnung}

1. Bei der Behandlung eines Anfangswertproblems für ein System von partiellen Differentialgleichungen erster Ordnung ist bekanntlich zuerst zu untersuchen, ob das Problem überhaupt Lösungen in der Umgebung der Anfangsmannigfaltigkeit zulässt und ob die Lösung durch die Anfangsbedingungen eindeutig bestimmt ist. Neben diesen Betrachtungen rein lokaler Natur erhebt sich die Frage, wie gross das Gebiet ist, in dem das Problem eine eindeutig bestimmte Lösung besitzt. Diese Frage sollte speziell in der heutigen Entwicklungslage der praktischen Mathematik beachtenswert sein, u.a. deshalb weil grosse elektronische Rechengeräte bei Behandlung der Systeme partieller Differentialgleichungen angewendet werden können. Im folgenden wird nun ein Versuch gemacht, Abschätzungen des Existenzgebietes der eindeutig bestimmten Lösung abzuleiten. Dabei wollen wir nach möglichst grosser Genauigkeit streben, weniger nach praktischer Einfachheit der Abschätzungen. Unsere Methode, die sich auch auf Systeme von impliziten partiellen Differentialgleichungen anwenden lässt, fusst auf dem von F. und R. Nevanlinna entwickelten koordinatenfreien Infinitesimalkalkül, auf der sog. absoluten Analysis ${ }^{1}$.

2. Hilfssatz über implizite Funktionen. Es sei $f(x, y)$ eine in den Kugeln $|x|<A_{x}(<\infty)$ und $|y|<A_{y}(<\infty)$ der Räume $R_{x}^{m}$ bzw. $R_{y}^{n}$ definierte Funktion mit Werten in dem $\operatorname{Raum} R_{z}^{n}(m, n<\infty)^{2}$. Wir betrachten die Gleichung

${ }^{1}$ In bezug auf die Grundbegriffe der absoluten Analysis verweisen wir auf die Monographie [5] der Herren Nevanlinna.

Aus der reichhaltigen Literatur zu der Theorie von Systemen partieller Differentialgleichungen seien hier die von Kamke [3] (S. 352-362) und Wazewski [8], [9] gegebenen Abschätzungen des Existenzgebietes erwähnt. Diese Verfasser haben die Frage in Koordinatenform betrachtet (die Differentialgleichung als nach einer partiellen Ableitung gelöst vorausgesetzt, eine Koordinatenhyperebene als Anfangsmannigfaltigkeit gewählt und die Existenz in einer Zone oder in einer Doppelpyramide bewiesen). Verallgemeinerungen dieser älteren Arbeiten auf Systeme partieller Differentialgleichungen sind in den letzten Jahren u.a. von Szarski [7] und Pawelski [6] gegeben worden

${ }^{2}$ Wir bezeichnen einen reellen linearen Raum mit $R_{u}=R_{u}^{p}$, wobei u die Variable 


$$
f(x, y)=0,
$$

und bestimmen unter gewissen Voraussetzungen ein Gebiet, in dem $y$ durch diese Gleichung als eine eindeutige Funktion von $x$ definiert wird. Daneben wird auch eine Abschätzung für die Funktion $y=y(x)$ gewonnen.

Es sei also angenommen:

1. Die Funktion $f(x, y)$ ist für $|x|<A_{x},|y|<A_{y}$ zweimal stetig differenzierbar.

2. $f(0,0)=0$.

3. inf $\left|f_{y}(0,0) d y\right|=\mu>0$, d.h. der Operator $f_{y}(0,0)$ ist regulär. $|d y|=1$

Wir definieren zwei stetige nichtnegative monoton wachsende Funktionen von zwei reellen Variablen $\varrho, r(\geqq 0)^{3}$

$$
\begin{aligned}
& M(\varrho, r)= \sup _{|x| \leqq \varrho}\left|f_{x}(x, y)\right|, \\
& \varphi(\varrho \mid \leqq r \\
&
\end{aligned}
$$

Über die Funktion $M(\varrho, r)$ sei noch zusätzlich angenommen:

4. $M(\varrho, 0)>0$, für $\varrho>0$.

Wegen der vorausgesetzten Stetigkeit von $f_{y}$ folgt $\varphi(\varrho, r)<\mu$ für genügend kleine $\varrho$ und $r$. Es sei $\varrho_{0}$ die obere Grenze derjenigen Zahlen $\varrho \leqq A_{x}$, für die $\lim _{r \rightarrow 0} \varphi(\varrho, r)<\mu$, und $r_{0}(\varrho)$ die obere Grenze derjenigen Zahlen $r \leqq A_{y}$, für die $\varphi(\varrho, r)$ mit festem $\varrho<\varrho_{0}$ kleiner als $\mu$ bleibt. Für $|x| \leqq \varrho<\varrho_{0},|y|<r<r_{0}(\varrho)$ ist

$$
\begin{gathered}
\inf _{|d y|=1}\left|f_{y}(x, y) d y\right| \geqq \inf _{|d y|=1}\left|f_{y}(0,0) d y\right|-\sup _{\substack{|x| \leqq \varrho \\
|y| \leqq r}}\left|f_{y}(x, y)-f_{y}(0,0)\right| \\
=\mu-\varphi(\varrho, r)>0 ;
\end{gathered}
$$

der Operator $f_{y}(x, y)$ ist also dann regulär.

Ist die Funktion $y=y(x)$ durch (1) definiert, so ergibt sich von (1) durch Differentiation nach $x$

$$
f_{x}(x, y) d x+f_{y}(x, y) y^{\prime}(x) d x=0
$$

oder, $f_{y}$ als regulär vorausgesetzt,

$$
d y=y^{\prime}(x) d x=-\left(f_{y}(x, y)\right)^{-1} f_{x}(x, y) d x,
$$

und $p$ die Dimension des Raumes angibt. Die Norm eines Vektors $u \in R_{u}$ im Sinne der in $R_{\boldsymbol{u}}$ eingeführten (etwa Minkowskischen) Metrik sei wie üblich mit $|u|$ bezeichnet. definiert.

${ }^{3}$ Die Norm $|A|$ einer linearen Abbildung $A: R_{\boldsymbol{x}} \rightarrow R_{\boldsymbol{y}}$ ist durch $|A|=\sup _{|\boldsymbol{h}|=1}|A h|$ 
und die Funktion $y=y(x)$ ist somit eine Lösung der Differentialgleichung (3). Ist umgekehrt $y=y(x)$ eine Lösung von (3), für die $y(0)=0$, so gilt wegen (2) $d f(x, y(x))=0$, und die Gleichung (1) wird identisch erfüllt.

Die Differentialgleichung (3) ist eine Gleichung von der Form

$$
d y=F(x, y) d x,
$$

wobei der Operator $F(x, y)=-\left(f_{y}(x, y)\right)^{-1} f_{x}(x, y)$ in einer konvexen Umgebung von $(0,0)$ im Produktraum $R_{x} \times R_{y}$ stetig differenzierbar ist. Die Theorie von Differentialgleichungen besagt ${ }^{4}$, dass eine Differentialgleichung dieser Art vollständig integrierbar ist, wenn die bekannte Integrabilitätsbedingung

$$
\left(F_{x}+F_{y} F\right) h k-\left(F_{x}-F_{y} F\right) k h=0 \quad\left(h, k \in R_{x}\right)
$$

für alle vorkommenden Werte $x, y$ besteht. Das ist aber hier gerade der Fall wegen der speziellen Form des Operators $F$. Die durch den Anfangspunkt $(0,0)$ gehende, eindeutig bestimmte Integralfläche existiert also bis zum Rand desjenigen konvexen Gebietes, in dem $F$ die erwähnte stetige Differenzierbarkeit besitzt. Die Aufgabe der Abschätzung des Existenzgebietes einer impliziten Funktion ist somit auf die Abschätzung desjenigen der Lösung einer Differentialgleichung erster Ordnung zurückgeführt worden.

Wir bezeichnen mit $\psi(\varrho, r)$ die nach den beiden Variablen monoton zuwachsende Funktion

$$
\psi(\varrho, r)=\frac{M(\varrho, r)}{\mu-\varphi(\varrho, r)},
$$

die für $\varrho<\varrho_{0}, r<r_{0}(\varrho)$ stückweise stetig differenzierbar ist. Der Differentialgleichung

$$
\frac{d r}{d \varrho}=\psi(\varrho, r)
$$

kann man eine der Bedingung $r(0)=0$ genügende Funktion $\bar{r}(\varrho)$ zuordnen, so dass sie die durch die Anfangsbedingung $r(0)=0$ eindeutig bestimmte Lösung dieser Differentialgleichung in dem Intervall $I=\left\{\varrho \mid 0 \leqq \varrho<\varrho_{0}, \bar{r}(\varrho)<r_{0}(\varrho)\right\}$ ist. Die Lösung $y=y(x)$ der Differentialgleichung (3) genügt nun der Ungleichung

$$
\left|y^{\prime}(x)\right| \leqq \psi(\varrho, r)
$$

4 Vgl. [5] und [3] S. 135 . 
auf der Kugel $\{x|| x|\leqq \varrho| y,(x) \mid \leqq r\}$ mit $\varrho<\varrho_{0}, r<r_{0}(\varrho)$. Daraus geht aber zugleich die Existenz der Funktion $y=y(x)$ in der Kugel $\{x|| x \mid \in I\}$ hervor.

Wir bezeichnen mit $\varrho=\bar{\varrho}(r)$ die inverse Funktion von $\bar{r}(\varrho)$; sie existiert in dem Gebiet $0 \leq r<r_{0}(\bar{\varrho})$, wobei $\bar{\varrho}$ die obere Grenze derjenigen Zahlen $\varrho<\varrho_{0}$ ist, für die $\bar{r}(\varrho)<r_{0}(\varrho)$. Die Funktion $\varrho=\bar{\varrho}(r)$ genügt dazu der Differentialgleichung

$$
d \varrho / d r=1 / \psi(\varrho, r) \equiv \chi(\varrho, r),
$$

wobei die Funktion $\chi$ nach den beiden Variablen stetig und monoton abnehmend ist.

Man erklärt die Funktion

$$
P(\varrho, r)=\int_{0}^{r} \chi(\varrho, r) d r
$$

für $\varrho<\varrho_{0}, r<r_{0}(\varrho)$ und definiert durch

$$
F(\varrho)=\int_{0}^{r_{0}(\varrho)} \chi(\varrho, r) d r=P\left(\varrho, r_{0}(\varrho)\right)
$$

eine monoton abnehmende Funktion für $0 \leq \varrho<\varrho_{0}$, die für $\varrho>\bar{\varrho}$ der Ungleichung

$$
F(\varrho) \leqq \varrho\left(r_{0}(\varrho)\right) \leqq \bar{\varrho}\left(r_{0}(\varrho)\right)<\varrho
$$

genügt. Bezeichnet man mit $\varrho_{x}$ die obere Grenze derjenigen Zahlen $\varrho<\varrho_{0}$, für die $F(\varrho)>\varrho$, so ist $\varrho_{x} \leqq \bar{Q}$, und die Funktion $r=\bar{r}(\varrho)$ existiert für $0 \leqq \varrho \leqq \varrho_{x}$. Das letzte Ergebnis heisst aber auch: die Funktion $y=y(x)$ existiert im Gebiet $|x|<\varrho_{x}$.

Es sei $r=G(\varrho)$ die eindeutige inverse Funktion ron $\varrho=\varrho(r)$ $=P\left(\varrho_{x}, r\right)$. Dann gilt für $x \leqq \varrho<\varrho_{x}$ die Abschätzung $\bar{r}(\varrho) \leqq G(\varrho)$, und es ist also

$$
y(x) \leqq \bar{r}(\varrho) \leqq G(\varrho) .
$$

Wir haben so den folgenden Hilfssatz 1 erreicht:

Unter den gemachten Annahmen 1-4 definiert die Gleichung (1) die Funktion $y=y(x) \quad(y(0)=0)$ in der Kugel $|x|<\varrho_{x}$, wobei $\varrho_{x}$ die obere Grenze derjenigen Zahlen $\varrho<\varrho_{0}$ bezeichnet, für die $P\left(\varrho, r_{0}(\varrho)\right)>\varrho$ gilt. Diese Funktion ist stetig differenzierbar und genügt der Abschätzung

$$
|y(x)| \leqq G(|x|) .
$$


Die erreichten Abschätzungen für das Existenzgebiet und für die Funktion $y(x)$ sind unter den gemachten Annahmen die bestmöglichen, d.h. es lassen sich Beispiele (etwa die Gleichung $f(x, y)=x^{2}+y^{2}-a y=0$, $a>0$ ) aufzeigen, bei denen diese Abschätzungen genau sind.

3. Hilfssatz über Differentialgleichungen. Betrachtet sei eine Differentialgleichung der Form

$$
d y=f(y) d t \quad\left(y, d y \in R_{y}^{n}, \quad d t \in R_{t}^{1}\right)
$$

wobei der Operator $f(y)$ folgende Eigenschaften hat:

1. $f(y)$ ist eine in der Kugel $\left|y-y_{0}\right| \leqq r_{y}(<\infty)$ des Raumes $R_{y}^{n}$ stetige Funktion von $y$ und erklärt für jedes $y$ dieser Kugel eine lineare Abbildung von $R_{t}^{1}$ in $R_{y}^{n}$.

2. $f(y)$ erfüllt die Lipschitz-Bedingung

$$
\left|f\left(y_{1}\right)-f\left(y_{2}\right)\right| \leqq M\left|y_{1}-y_{2}\right|
$$

für je zwei Punkte $y_{1}, y_{2}$ in der Kugel $\mid y-y_{0} \leqq r_{y}$.

In Koordinatenform entspricht der Differentialgleichung (4) ein Normalsystem

$$
d y_{i} / d t=f_{i}\left(y_{1}, y_{2}, \ldots, y_{n}\right) \quad(i=1,2, \ldots, n) .
$$

Gemäss der Theorie von Differentialgleichungen ${ }^{5}$ hat obige Gleichung (oder obiges Gleichungssystem) eine und nur eine Lösungskurve $y=Y(t)$, die durch den Punkt $\left(t_{0}, y_{0}\right)$ geht, und diese Kurve existiert bis zum Rande des Zylindergebietes

$$
\left|y-y_{0}\right|<r_{y}, \quad|t|<\infty,
$$

in $R_{t} \times R_{y}$, wo der Operator $f$ definiert ist. Wir wollen das Existenzgebiet für die Lösung unter der Anfangsbedingung $Y\left(t_{0}\right)=y_{0}$ abschätzen, indem wir eine Abschätzung für die Norm $\left|Y(t)-y_{0}\right|$ bilden und darauf achten, wo diese kleiner als $r_{y}$ bleibt.

Es sei $F(r)$ eine in dem Intervall $0 \leqq r<r_{y}$ definierte, stetige reelle Funktion, die

a) für $r \geqq\left|y-y_{0}\right|$ der Ungleichung $F(r) \geqq|f(y)|$ genügt,

b) für $0 \leqq r_{1}, r_{2}<r_{y}$ eine Lipschitz-Bedingung erfüllt:

$$
\left|F\left(r_{1}\right)-F\left(r_{2}\right)\right| \leqq \bar{M}\left|r_{1}-r_{2}\right|
$$

Speziell kann z.B.

$$
F(r)=\sup _{\left|y-y_{0}\right| \leqq r}|f(y)|
$$

gesetzt werden.

5 Vgl. z.B. [3] S. 135. 
Die Differentialgleichung

$$
d s=F(s) d t
$$

hat in einer Strecke rechts von $t_{0}$ eine und nur eine Lösung $s=S(t)$ unter der Anfangsbedingung $S\left(t_{0}\right)=0$. Wird die eindeutige inverse Funktion von $S(t)$ mit $t=T(s)$ bezeichnet, so ist für $0 \leqq r<r_{y}$

$$
T(r)=t_{0}+\int_{0}^{r} \frac{d s}{F(s)} .
$$

Die Lösung $s=S(t)$ existiert, solange $0 \leqq S(t)<r_{y}$ ist, also für $t_{0} \leqq t<t_{0}+\varrho_{t}$, wobei

$$
\varrho_{t}=T\left(r_{y}\right)-t_{0}=\int_{0} \frac{r_{y}}{d s} \frac{}{F(s)} .
$$

Sei $\left[t_{0}, t_{1}\right]$ ein Intervall rechts von $t_{0}$, in dem $Y(t)$ und $S(t)$ existieren. Die Funktion $\varphi(t)=\left|Y(t)-y_{0}\right|$ ist dort stückweise stetig differenzierbar, und es gilt die Ungleichung

$$
\varphi^{\prime}(t) \leqq\left|Y^{\prime}(t)\right|=|f(Y(t))| \leqq F\left(Y(t)-y_{0}\right)=F(\varphi) .
$$

Es sind $\varphi(t)$ bzw. $S(t)$ für $0 \leqq t \leqq t_{1}$ Lösungen der Differentialgleichungen

$$
\varphi^{\prime}(t)=G(\varphi) \quad \text { bzw. } \quad S^{\prime}(t)=F(S)
$$

unter der Anfangsbedingung

$$
\varphi\left(t_{0}\right)=S\left(t_{0}\right)=0,
$$

wobei die rechten Seiten $G(u)$ und $F(u)$ in der Beziehung $G(u) \leqq F(u)$ stehen. Daher schliesst man: $\varphi(t) \leqq S(t)$ für $t_{0} \leqq t \leqq t_{1}{ }^{6}$. Das bedeutet, die Funktion $\varphi(t)$ existiert in dem ganzen Intervall $t_{0} \leqq t<t_{0}+\varrho_{t}$, oder mit Rücksicht auf Obiges, dass $Y(t)$ für $\left|t-t_{0}\right|<\varrho_{t}$ existiert.

Wir haben somit den folgenden Hilfssatz 2:

Es sei $f(y)$ eine lineare Operatorfunktion mit den Eigenschaften 1, 2 und $F(r)$ eine in dem Intervall $0 \leqq r<r_{y}$ definierte stetige reelle Funktion mit den Eigenschaften a, b. Dann hat die Differentialgleichung (4) unter der Anfangsbedingung $y\left(t_{0}\right)=y_{0}$ eine und nur eine Lösung, in deren Existenzintervall wenigstens die Strecke

eingeht.

$$
\left|t-t_{0}\right|<\varrho_{t}=\int_{0}^{r_{y}} \frac{d s}{F(s)}
$$

6 Vgl. [3] S. 91. 
Die spezielle Wahl (5) der Funktion $F(r)$ ergibt die genaueste Abschätzung, die unter den gemachten Annahmen nicht zu vergrössern ist. Dies geht aus dem Beispiel

$$
f(y)=\frac{y}{|y|} \frac{1}{r_{y}-|y|}
$$

hervor.

4. Nach den obigen Vorbereitungen wollen wir zu dem eigentlichen Gegenstand dieser Arbeit übergehen. Wir betrachten eine implizite Differentialgleichung erster Ordnung,

$$
H(x, d z / d x, z)=0 \quad\left(x \in R_{x}^{m}, z \in R_{z}^{1}, H \in R_{H}^{p}, 1 \leqq p<m\right),
$$

nebst einer gegebenen "Anfangsmannigfaltigkeit» $x=\xi(t)\left(t \in R_{t}^{m-p}\right)$ und einer "Anfangswertfunktion» $z=\zeta(t)$. Falls nun solche Elemente $x_{0}=\xi\left(t_{0}\right), z_{0}=\zeta\left(t_{0}\right), y_{0} \in R_{y}^{m}=R_{x^{*}}$ existieren 7 , bei denen $H\left(x_{0}, y_{0}, z_{0}\right)=0$, $\zeta^{\prime}\left(t_{0}\right)=y_{0} \xi^{\prime}\left(t_{0}\right)$ ist, und falls die Funktionen $H, \xi, \zeta$ gewisse weitere Voraussetzungen erfüllen, so ist bekanntlich in der Umgebung von $x_{0}$ die Existenz einer eindeutig bestimmten Funktion $z=z(x)$ garantiert, die der Differentialgleichung (6) und der Gleichung $z(\xi(t))=\zeta(t)$ genügt $^{8}$. Wir wollen im folgenden das Existenzgebiet dieser Funktion abschätzen, nachdem wir die Annahmen der Einfachheit halber folgendermassen formuliert haben:

Es seien $R_{u}^{p}$ und $R_{v}^{m-p}$ zwei orthogonale Komplemente des euklidisch metrisierten Argumentraums $R_{x}^{m}$. Dementsprechend zerfällt dann der zu $R_{x}$ duale Raum $R_{y}^{m}$, bezüglich der von der $R_{x}$-Metrik induzierten dualen Metrik, in zwei Orthogonalkomplemente $R_{f}^{p}$ und $R_{g}^{m-p}$, so dass $g u=f v=0$ für $g \in R_{g}, f \in R_{f}, u \in R_{u}, v \in R_{v}$. Für die Funktion $H(u, v, f, g, z)$, deren Werte in $R_{H}^{p}$ liegen, wird folgendes angenommen:

1. $H(u, v, f, g, z)$ ist in dem Gebiet $U:\left|u-u_{0}\right|<A_{u},\left|v-v_{0}\right|<A_{v}$, $\left|f-f_{0}\right|<A_{f},\left|g-g_{0}\right|<A_{g},\left|z-z_{0}\right|<A_{z} \quad$ zweimal stetig differenzierbar und $H\left(u_{0}, v_{0}, f_{0}, g_{0}, z_{0}\right)=0$.

2. $H_{f}\left(u_{0}, v_{0}, f_{0}, g_{0}, z_{0}\right)$ ist eine reguläre lineare Abbildung von $R_{f}$ auf $R_{H}$.

3. Für alle Elemente $(u, v, f, g, z)$ in $U$, die der Gleichung $H(u, v, f, g, z)=0$ genügen, gilt die »Integrabilitätsbedingung»

$$
R h k=0 \quad\left(h, k \in R_{t}=R_{H^{*}}\right),
$$

wobei $R=R(u, v, f, g, z)$ den durch

' Unter dem zu $R_{x}$ dualen Raum $R_{y}$ der auch mit $R_{x^{*}}$ bezeichnet wird, versteht man den Raum der in $R_{\boldsymbol{x}}$ definierten reellen linearen Funktionen.

8 Vgl. z.B. [2] S. 53-68; in [4] wird auch dasselbe Resultat in der hier benutzten "absoluten" Schreibweise abgeleitet. 


$$
R h k=\wedge\left\{\left(H_{u}^{*}+f H_{z}^{*}\right) h H_{f}^{*} k+\left(H_{v}^{*}+g H_{z}^{*}\right) h H_{g}^{*} k\right\}
$$

definierten alternierenden bilinearen Operator bezeichnet ${ }^{9}$.

Wit setzen im besonderen voraus, dass die Anfangsfläche, in der die Funktionswerte gegeben sind, eine zu $R_{v}$ parallele $(m-p)$-dimensionale Hyperebene ist. Dann ist in den Punkten dieser Fläche $u=u_{0}$ (= konst.) und die Variable $v$ kann als Parameter gewählt werden. Die gegebenen Funktionswerte bezeichne man mit $\zeta(v)$ und nehme folgendes an:

4. $z=\zeta(v)$ ist für $\left|v-v_{0}\right|<A_{v}$ eine dreimal stetig differenzierbare Funktion, für die $\left|\zeta(v)-z_{0}\right|<A_{z},\left|\zeta^{\prime}(v)-g_{0}\right|<A_{g}$.

5. $\zeta\left(v_{0}\right)=z_{0}, \quad \zeta^{\prime}\left(v_{0}\right)=g_{0}$.

Es existiert also, wie schon bemerkt, in der Umgebung von $x_{0}=\left(u_{0}, v_{0}\right)$ eine eindeutig bestimmte Funktion $z=z(x)=z(u, v)$, die die Gleichung (6) und die Bedingung $z\left(u_{0}, v\right)=\zeta(v)$ erfüllt. Bei Abschätzung des Existenzgebietes dieser Funktion wollen wir die oben gegebenen Hilfssätze sowie den Umkehrsatz von Nevanlinna [5] und Bartle [1] benutzen. Wir gehen aus von der Gleichung

$$
H\left(u_{0}, v, f, \zeta^{\prime}(v), \zeta(v)\right)=0,
$$

die die Funktion $f=f(v)$ in einer Umgebung von $v_{0}$ bestimmt, und wollen mittels des Hilfssatzes 1 diese Umgebung abschätzen. Darauf bildet man die charakteristischen Differentialgleichungen

$$
\begin{array}{ll}
d u=H_{f}^{*} d t, \quad d v=H_{g}^{*} d t, & d f=\left(-H_{u}^{*}-H_{z}^{*} f\right) d t, \\
d g=\left(-H_{v}^{*}-H_{z}^{*} g\right) d t, & d z=\left(f H_{f}^{*}+g H_{g}^{*}\right) d t,
\end{array}
$$

die sich für den Produktraum $R_{\Omega}=R_{u} \times R_{v} \times R_{f} \times R_{g} \times R_{z}$ auch in der Form

$$
d \Omega=\Phi(\Omega) d t
$$

zusammenfassen lassen. Dieses System hat in der Umgebung des Nullpunktes ein Integral $\Omega(t)=\Omega(t ; \bar{\Omega})$, das durch die Anfangsbedingung

$$
\Omega(0)=\bar{\Omega}=\left(u_{0}, v, f(v), \zeta^{\prime}(v), \zeta(v)\right)
$$

(mit festem $v$ ) eindeutig bestimmt ist, vorausgesetzt, dass der Punkt $\bar{\Omega}$ zu dem Gebiet $U$ gehört. Ein Existenzgebiet für dieses Integral ergibt sich durch Hilfssatz 2. Die Lösung von (8) unter der Anfangsbedingung (9) definiert in dem Produktraum $R_{\omega}=R_{v} \times R_{t}$ in der Umgebung von $\omega_{0}=\left(v_{0}, t_{0}\right)$ eine eindeutige differenzierbare Funktion

$$
\text { (10) } \Omega=\Omega(\omega)=\Omega(v, t)=(U(v, t), V(v, t), F(v, t), G(v, t), Z(v, t)) \text {, }
$$

9 Einer linearen Abbildung $A: R_{u} \rightarrow R_{x}$ ordnet man durch $x^{*}(A u)=\left(A^{*} x^{*}\right) u$ $\left(u \in R_{u}, x^{*} \in R_{x^{*}}\right)$ eine eindeutig bestimmte duale Abbildung $A^{*}: R_{x^{*}} \rightarrow R_{u^{*}}$ zu. 
die in einer Umgebung von $\omega_{0}$ umkehrbar ist. Durch Einführung geeigneter Hilfsmetriken in $R_{\omega}$ und $R_{\Omega}$ lässt sich das Existenzgebiet der Umkehrfunktion durch den erwähnten Satz abschätzen, wobei zugleich eine Abschätzung für das Existenzgebiet der Lösung der partiellen Differentialgleichung (6) gewonnen wird.

Wir kommen also auf die Gleichung (7) und auf die zueinander dual metrisierten Räume $R_{f}$ und $R_{u}$ bzw. $R_{g}$ und $R_{v}$ zurück. Der Hilfssatz 1 ist nun anwendbar, wobei die Konstante $\mu$ den Wert

$$
\mu_{0}=\inf _{|d f|=1}\left|H_{f}\left(u_{0}, v_{0}, f_{0}, g_{0}, z_{0}\right) d f\right|
$$

hat, und weiter

$$
\begin{gathered}
M(\varrho, r)=\sup _{\substack{\left|v-v_{0}\right| \leqq \varrho \\
\left|f-f_{0}\right| \leqq r}}\left|H_{v}+H_{g} \zeta^{\prime \prime}+H_{z} \zeta^{\prime}\right| \quad\left(u=u_{0}, g=\zeta^{\prime}(v), z=\zeta(v)\right), \\
\varphi(\varrho, r)=\sup _{\substack{v-v_{0}|\leqq \varrho\\
| f-f_{0} \mid \leqq r}}\left|H_{f}\left(u_{0}, v, f, \zeta^{\prime}(v), \zeta(v)\right)-H_{f}\left(u_{0}, v_{0}, f_{0}, g_{0}, z_{0}\right)\right|
\end{gathered}
$$

sind. Bei Anwendung der im Hilfssatz benutzten Bezeichnung $\psi(\varrho, r)$ kann das Ergebnis jetzt wie folgt ausgedrückt werden:

Die Funktion $f=f(v)$ existiert in dem Gebiet $\left|v-v_{0}\right|<\varrho_{v}$, wobei

$$
\varrho_{v}=\sup _{\varrho \in B} \varrho \quad\left(B=\left\{\varrho \mid \int_{0}^{\left.r_{0} \varrho\right)} \frac{d r}{\psi(\varrho, r)}>\varrho\right\}\right) .
$$

Für $\left|v-v_{0}\right| \leqq \varrho<\varrho_{v}$ gilt

$$
\left|f(v)-f_{0}\right| \leqq G(\varrho)
$$

worin $G(\varrho)$ die in Hilfssatz 1 erklärte inverse Funktion von $\varrho=\varrho(r)$ $=P\left(\varrho_{v}, r\right)$ bezeichnet.

Zweitens schätzen wir das Existenzgebiet der Lösung von charakteristischen Differentialgleichungen ab, die bei gewisser Parameterwahl die Form (8) haben. Wir führen in den Raum $R_{\Omega}^{2 m+1}$ eine Minkowskische Hilfsmetrik ein, indem wir für die Strecke $d \Omega$ mit den Komponenten $d u$, $d v$, $d f, d g, d z$ eine Norm

$$
|d \Omega|=\max \left(\frac{|d u|}{A_{u}}, \frac{|d v|}{A_{v}}, \frac{|d f|}{A_{f}}, \frac{|d g|}{A_{g}}, \frac{|d z|}{A_{z}}\right)
$$

definieren. Wenn $\bar{\Omega}$ zu dem Gebiet $U$ gehört, wie im folgenden vorausgesetzt wird, liegt die Kugel

$$
|\Omega-\bar{\Omega}|<1-\left|\bar{\Omega}-\Omega_{0}\right|=1-\max \left\{\frac{\left|v-v_{0}\right|}{A_{v}}, \frac{\left|f(v)-f_{0}\right|}{A_{f}}, \frac{\left|\zeta^{\prime}(v)-g_{0}\right|}{A_{g}}, \frac{\left|\zeta(v)-z_{0}\right|}{A_{z}}\right\}
$$

ganz in dem Gebiet $U$. Wir bezeichnen 


$$
\begin{gathered}
\sigma(\Omega)=\sigma(u, v, f, g, z)=\max \left\{\frac{\left|H_{f}\right|}{A_{u}}, \frac{\left|H_{g}\right|}{A_{v}}, \frac{\left|H_{u}+H_{z} f\right|}{A_{f}}, \frac{\left|H_{v}+H_{z} g\right|}{A_{s}}, \frac{\left|H_{f} f+H_{g} g\right|}{A_{z}}\right\}, \\
F(\lambda)=\sup _{\left|\Omega-\Omega_{0}\right| \leqq \lambda}(\sigma(\Omega)), \\
T(\lambda)=\int_{0}^{\lambda} \frac{d \lambda}{F(\lambda)}
\end{gathered}
$$

und die eindeutige inverse Funktion der letzten mit

$$
\lambda=\Lambda(t),
$$

so dass u.a. $|\Phi(\Omega)| \leqq F(\lambda)$ für $\left|\Omega-\Omega_{0}\right| \leqq \lambda$ gilt. Der Hilfssatz 1 ist nun auf die Funktionen $\Phi(\Omega)$ und $F(\lambda)$ anwendbar und liefert somit für das Existenzgebiet der Lösung von (8) eine Abschätzung:

$$
\left|t-t_{0}\right|<\underline{o}_{t}=\int_{\left|\bar{\Omega}-\Omega_{0}\right|}^{1} \frac{d \lambda}{F(\lambda)}=T(1)-T\left(\left|\bar{\Omega}-\Omega_{0}\right|\right) .
$$

Man bezeichne

$$
\lambda(\varrho)=\sup _{\left|v-v_{0}\right| \leqq \varrho}\left(\frac{\varrho}{A_{v}}, \frac{\left|\zeta^{\prime}(v)-g_{0}\right|}{A_{g}}, \frac{G(\varrho)}{A_{f}}, \frac{\left|\zeta(v)-z_{0}\right|}{A_{z}}\right),
$$

so dass

$$
\bar{\Omega}-\Omega_{0} \leqq \lambda\left(\mid v-v_{0}\right)
$$

Die Funktion (10) ist also in dem Gebiet

$$
\left|v-v_{0}\right|<\varrho_{v}, \quad \mid t-t_{0}<\int_{i\left(v-v_{0}\right)} \frac{d \lambda}{F\left(\lambda_{0}\right)}
$$

definiert und in den beiden Variablen sogar stetig differenzierbar.

Um die zu betrachtende partielle Differentialgleichung (6) zu integrieren, ist die Variable $\omega=(v, t)=\omega(x)$ aus der Gleichung $x=X(v, t)$ $=(U(v, t), V(v, t))$ zu lösen und danach in die Gleichung $z=Z(v, t)$ zu substituieren. Das Gebiet, in dem diese Lösung möglich ist, kann durch den Umkehrsatz abgeschätzt werden, nachdem in $R_{\omega}$ eine Hilfsmetrik mit der Norm

$$
|d \omega|=\max \left(d v, d t \mid / \mu_{0}\right)
$$

eingeführt worden ist, in der

$$
\mu_{0}=\inf _{|d f|=1}\left|H_{f}\left(u_{0}, v_{0}, f_{0}, g_{0}, z_{0}\right) d f\right| .
$$


Bei dieser Wahl der Hilfsmetrik hat die Konstante $\mu$ des Umkehrsatzes den Wert $\mu=1$. Um die Funktion

$$
\varphi(r)=\sup _{\left|\omega-\omega_{0}\right| \leqq r}\left|X^{\prime}(\omega)-X^{\prime}\left(\omega_{0}\right)\right|=\sup _{\substack{\left|\omega-\omega_{0}\right| \leqq r \\|d \omega| \mid=1}}\left|\left(X^{\prime}(\omega)-X^{\prime}\left(\omega_{0}\right)\right) d \omega\right|
$$

weiter abzuschätzen, setze man

$$
K(\lambda)=\sup _{\left|\Omega-\Omega_{0}\right| \leqq \lambda} \sqrt{\left|H_{f}(\Omega)-H_{f}\left(\Omega_{0}\right)\right|^{2}+\left|H_{g}(\Omega)-H_{g}\left(\Omega_{0}\right)\right|^{2}} .
$$

Es gilt dann

$$
\begin{aligned}
\varphi(r) & \leqq \sup _{\left|\omega-\omega_{0}\right| \leqq r}\left(\left|X_{t}(\omega)-X_{t}\left(\omega_{0}\right)\right|+\left|X_{v}(\omega)-X_{v}\left(\omega_{0}\right)\right|\right) \\
& =\sup _{\left|\omega-\omega_{0}\right| \leqq r} \sqrt{\left|U_{t}(\omega)-U_{t}\left(\omega_{0}\right)^{2}+\right| V_{t}(\omega)-\left.V_{t}\left(\omega_{0}\right)\right|^{2}} \\
& +\sup _{\left|\omega-\omega_{0}\right| \leqq r} \sqrt{\left|U_{v}(\omega)-U_{v}\left(\omega_{0}\right)\right|^{2}+\left|V_{v}(\omega)-V_{v}\left(\omega_{0}\right)\right|^{2}} \\
& \leqq \sup _{\left|\omega-\omega_{0}\right| \leqq r} \sqrt{\left|H_{f}(\Omega(\omega))-H_{f}\left(\Omega_{0}\right)^{2}+\right| H_{g}(\Omega(\omega))-\left.H_{g}\left(\Omega_{0}\right)\right|^{2}} \\
& +\sqrt{2} \sup _{\left|\omega-\omega_{0}\right| \leqq r}\left|\left(\frac{\partial \Omega(t ; \bar{\Omega}(v))}{\partial \bar{\Omega}}-\frac{\partial \Omega(0 ; \bar{\Omega}(v))}{\partial \bar{\Omega}}\right)\right| \\
& \leqq K\left(\Lambda\left(T(\lambda(r))+\mu_{0} r\right)\right)+\sqrt{2}\left(e^{\int_{0}^{\mu_{0} r} k(\tau, \lambda(r)) d \tau}-1\right)
\end{aligned}
$$

wobei

bezeichnet.

$$
k(\tau, \lambda)=\sup _{\left|\Omega-\Omega_{0}\right| \leqq A(T(\lambda)+\tau)}\left|\Phi^{\prime}(\Omega)\right|
$$

Nach dem Umkehrsatz in [1] existiert somit die eindeutige Funktion $\omega=\omega(x)$ in der Kugel

$$
\left|x-x_{0}\right|<R=\int_{0}^{r_{0}}\left(\mu_{0}-\varphi(\varrho)\right) d \varrho,
$$

deren Radius sich wie folgt abschätzen lässt:

$$
R \geqq \bar{R}=\int_{0}^{r_{0}}\left(1-K\left(\Lambda\left(T(\lambda(\varrho))+\mu_{0} \varrho\right)\right)-\sqrt{2}\left(e^{\int_{0}^{\mu_{0} \varrho} k(\tau, \lambda(\varrho)) d \tau}-1\right)\right) d \varrho,
$$

wobei $r_{0}$ die obere Grenze derjenigen Zahlen $r\left(<r_{1}\right)$ bezeichnet, für die $\varphi(r)<1$ ist. Hierbei ist $r_{1}$ der Radius der grössten $R_{\omega}$-Kugel im Gebiet (11), also die Wurzel der Gleichung 


$$
\mu_{0} r=\int_{\lambda(r)}^{1} \frac{d \lambda}{F(\lambda)}
$$

Die Kugel (12) ist also in dem Existenzgebiet der Lösung der Differentialgleichung (6) enthalten, und $\bar{R}$ dient folglich auch zur Abschätzung des letzteren.

5. Die obigen Ergebnisse sind in einer sehr allgemeinen Form angegeben. Um eine konkretere Darstellung der Methode vorzulegen, wollen wir die spezielle partielle Differentialgleichung

$$
f g=\frac{2 u z\left(1+v^{2}\right)}{\left(1-u^{2}\right)^{2}\left(1-v^{2}\right)^{2}}\left(u, v \text { reelle Variablen; } f=\frac{\partial z}{\partial u}, g=\frac{\partial z}{\partial v}\right),
$$

betrachten und das Existenzgebiet der durch die Anfangskurve $u=0$, $z=\frac{v}{1-v^{2}}$ gehenden Lösung abschätzen. Dabei werden wir auch die Genauigkeit der Abschätzung erfahren, denn die explizite Lösung der Differentialgleichung unter der erwähnten Anfangsbedingung ist

$$
z=\frac{v}{\left(1-u^{2}\right)\left(1-v^{2}\right)},
$$

welche Funktion das exakte Existenzgebiet $|u|<1,|v|<1$ hat.

Die Funktion $f=f(v)$ unserer allgemeinen Betrachtung ist hier wegen der speziellen Form der Differentialgleichung explizit auflösbar, und zwar ist $f(v) \equiv 0$, so dass in der Funktion $\lambda(\varrho)$ auch $G(\varrho) \equiv 0$ gesetzt werden kann.

Man wählt $A_{u}=A_{v}=\delta<1, \quad A_{f}=A_{g}=A \geqq \frac{1+\delta^{2}}{\left(1-\delta^{2}\right)^{2}}$ und hat also $F(\lambda)=\max F_{i}(\lambda)(i=1,2,3,4)$,

wobei

$$
\begin{aligned}
& F_{1}(\lambda)=\frac{1+A \lambda}{\delta}, \quad F_{2}(\lambda)=2 \lambda(1+A \lambda), \\
& F_{3}(\lambda)=\frac{2 \lambda\left(1+\delta^{2} \lambda^{2}\right)\left(1+\delta \lambda+3 \delta^{2} \lambda^{2}-\delta^{3} \lambda^{3}\right)}{\left(1-\delta^{2} \lambda^{2}\right)^{5}}, \\
& F_{4}(\lambda)=\frac{2 \delta \lambda}{\left(1-\delta^{2} \lambda^{2}\right)^{5}}\left(2 \delta \hat{\lambda}^{2}\left(3+\delta^{2} \lambda^{2}\right)+\left(1-\delta^{4} \lambda^{4}\right)\left(\lambda+A^{-1}\right) .\right.
\end{aligned}
$$

Weiter hat man

$$
\lambda(\varrho)=\max \left(\frac{\varrho}{\delta}, \frac{\varrho^{2}\left(3-\varrho^{2}\right)}{A\left(1-\varrho^{2}\right)^{2}}, \frac{\varrho}{A\left(1-\varrho^{2}\right)}\right),
$$


und

$$
k(\tau, \lambda)=S(\Lambda(T(\lambda)+\tau)), \operatorname{mit} S(\lambda)=\max \left(\frac{A}{\delta}, 2+4 A \lambda, W(\delta \lambda)\right),
$$

wobei $W(t)$

$=\frac{2}{A\left(1-t^{2}\right)^{5}}\left(\delta t\left(1-t^{4}\right)+(1+A t)\left(1+10 t^{2}+5 t^{4}\right)+\frac{2 t^{2}}{1-t^{2}} \max \left(w_{1}(t), w_{2}(t)\right)\right)$

mit

$$
\begin{aligned}
& w_{1}(t)=2 A\left(3+14 t^{2}+3 t^{4}\right)+\delta\left(3-2 t^{2}-t^{4}\right), \\
& w_{2}(t)=A\left(9+22 t^{2}+9 t^{4}\right)-\delta\left(3-2 t^{2}-t^{4}\right) .
\end{aligned}
$$

Je nach der anpassenden Wahl der Konstante $\delta, A$ kann nun nach unserer Methode eine Abschätzung des Existenzradius ausgerechnet werden; die grösste Abschäłzung $R=0,026$ ergibt sich mit $\delta=0,39$, $A=1,60 .{ }^{10}$

Die übliche Wazewskische Abschätzung gibt, angewandt auf das obige Beispiel, ein Existenzgebiet

$$
|u|<7 \cdot 10^{-7},|v|<0,0075-2,3 \cdot|u| \text {. }
$$

6. Wir haben in den obigen Betrachtungen vorausgesetzt, dass die Werte der Lösung $z=z(x)$ auf einer $(m-p)$-dimensionalen Hyperebene vorgeschrieben sind. Dies bedeutet jedoch keine Einschränkung. Denn, es sei vielmehr angenommen, dass die Funktionswerte auf einer beliebigen, dreimal stetig differenzierbaren $(m-p)$-dimensionalen durch den Punkt $x_{0}$ gehenden Fläche gegeben sind. Wählt man dann den Unterraum $R_{v}$ parallel zu der Tangentialebene in Punkt $x_{0}$, so kann diese Fläche in der Form $u=u_{0}+\gamma(v)$ ausgedrückt werden, wobei die Funktion $\gamma(v)$ in einer Umgebung von $v_{0}$ eindeutig und dreimal stetig differenzierbar ist $\left(\gamma\left(v_{0}\right)=0, \gamma^{\prime}\left(v_{0}\right)=0\right)$. Die Transformation $u=\bar{u}+\gamma(\bar{v}), v=\bar{v}$ führt die Glejchung $H(u, v, f, g, z)=0$ in die Gleichung

$$
H\left(\bar{u}+\gamma(\bar{v}), \bar{v}, \bar{f}, \bar{g}+\bar{f}_{\gamma^{\prime}}(\bar{v}), \bar{z}\right)=\bar{H}(\bar{u}, \bar{v}, \bar{f}, \bar{g}, \bar{z})=0
$$

über, deren Lösung $\bar{z}(\bar{u}, \bar{v})$ nun auf der Hyperebene $\bar{u}=u_{0}$ vorgeschriebene Werte hat. Somit ist die Aufgabe auf den schon betrachteten Sonderfall zurückgeführt.

10 Mit Dankbarkeit sei erwähnt, dass dieses Ergebnis von Herrn Erkki Mikkola, cand. rer. nat. (Abteilung für angewandte Mathematik, Universität Helsinki), mit IBM 1620 ausgerechnet worden ist. 


\section{Literatur}

[1] Bartle, R. G.: On the opennes and inversion of differentiable mappings. - Ann. Acad. Scient. Fennicæ A. I. 257, 1958, 8 S.

[2] Charathéodory, C.: Variationsrechnung und partielle Differentialgleichungen erster Ordnung, I, - 2. Aufl., Teubner, Leipzig, 1956, XII + 171 S.

[3] Kaмke, E.: Differentialgleichungen reeller Funktionen. - Akademische Verlagsges., Leipzig, 1930, XIV + $436 \mathrm{~S}$.

[4] Manninen, J.: Zur Charakteristikentheorie von Systemen partieller Differentialgleichungen erster Ordnung. - Ann. Acad. Scient. Fennicæ A. I. 283, 1960, $37 \mathrm{~S}$.

[5] Nevanlinna, F. und R.: Absolute Analysis. - Grundlehren math. Wiss. 102, Springer, Berlin-Göttingen-Heidelberg, 1959, VIII - $259 \mathrm{~S}$.

[6] Pawelski, W.: Appréciation du domaine d'existence de l'intéz̧rale d'un système involutif d'équations aux dérivées partielles du premier ordre. - Ann. Polon. Math. II. 1, 1955, S. 29-36.

[7] Szarski, J.: Sur un système d'équations aux dérivées partielles du premier ordre complètement intégrable. - Ann. Soc. Polon. Math. 24, 1953, S. 9-16.

[8] Ważewski, T.: Sur le domaine d'existence des intégrales de l'équation aux dérivées partielles du premier ordre. - Ann. Soc. Polon. Math. 13, 1934, S. $1-9$.

[9] -»- Sur l'appréciation du domaine d'existence des intégrales de l'équation aux dérivées partielles du premier ordre. - Ann. Soc. Polon. Math. 14, 1935 , S. $149-177$. 